\title{
Eye Disease Classification based on Deep Belief Networks
}

\author{
K.G. Rani Roopha Devi, R. Mahendra Chozhan
}

\begin{abstract}
Problems affecting the eye can range from infections of the eye or the eyelid, genetically inherited eye problems, eye injuries or objects in the eye, and eye disorders that are the result of conditions that affect many organs. While classification of disease stages is critical to understanding disease risk and progression, several systems based on red tissues eye photographs are known. Most of these require in-depth and time-consuming analysis of eye images. Herein, we present an automated computer-based classification algorithm using Deep belief networks (DBNs), as well as network initialization effect to classify isolated events. Classification is a data mining (machine learning) technique used to predict group membership for data instances. In Image classification analyzes the numerical properties of various image features and organizes data into categories. To simplify the problems of prediction or classification, neural networks are being introduced. In this paper, the introduction of Deep- belief network $(D B N)$ to classify the types of diseases affects the human eye. The DBN classify the three types of diseases such as defected eye, conjunctivitis, Keratoconus, and other types of diseases. The eye diseases features are compared with the features of the database. Finally the classification output produces the types of diseases present in the eye image. The implementations are done using the Matlabsoftware. Finally, the classification performances of $D B N$ architecture were analyzed using the accuracy, precision and recall parameters etc.
\end{abstract}

IndexTerms-Eye diseases, Deep belief networks, classifier, image classification, feature extraction.

\section{INTRODUCTION}

The human eye is an organ which responds to light and pressure. As a sense organ, the mammalian eye permits vision. Human eyes help to give a three dimensional, moving picture, ordinarily shaded in daylight. For the most part the ruddiness spot happen in the eye changes its typical vision. Ordinarily the human eye is distinguished the event of issues. The idea of the abandoned part and the kinds of ailments influenced can be ordered utilizing the sort of classifier. Here there is requirement for computerized reasoning for the grouping forms neural system. The machine learning method and the classifier calculation distinguishes and the recognize the eye sicknesses [1]. In machine learning, a deep belief network (DBN) is a generative graphical model, or on the other hand a class of deep neural network, made out of numerous layers of dormant factors ("hidden units"), with associations between the layers however not between units inside each layer. The PC variant assumes the real job in the advanced image preparing strategies. The image

Revised Version Manuscript Received on 10, September 2019.

K.G. Rani Roopha Devi, Ph.D Scholar, Madurai Kamaraj University, Madurai, Tamil Nadu, India.

R. Mahendra Chozhan, Chozhan Dental Clinic, Kodaikanal, Periyakulam, Lakshmipuram Tamil Nadu, India. segmentation, classification, contrast enhancement and so forward, apply to the eye image for future process [2]. A few DL designs have been accounted for in the writing, including deep neural network (DNN), RNN, convolution neural network (CNN), deep auto encoder (DA), deep Boltzmann machine (DBM), deep belief network (DBN), deep lingering network, deep convolution opposite illustrations network, and so on [3]. Classification can be performed on organized or unstructured information. Classification is where we classify information into a given number of classes. The fundamental objective of a classification issue is to distinguish the classification/class to which information will fall under. Anyway in the strategic relapse works just when the anticipated variable is twofold, accept all indicators are autonomous of one another, and expect information is free of missing qualities. So the classification execution issue can be overwhelmed by the presentation of deep belief network (DBN). The session 2 incorporates the writing study for the fundamental classification procedures. The session 3 incorporates the proposed framework and the end part in the segment 4 . The following area pursues the literature survey.

\section{LITERATURE SURVEY}

Yi Qin et al., [4] proposed an insightful and incorporated methodology dependent on deep belief networks (DBNs), enhanced strategic Sigmoid (I sigmoid) units and imprudent highlights. Back-spread is an established strategy ordinarily dependent on angle plummet improvement calculation to refresh the loads and predisposition, which is broadly utilized in different machine learning models.

GongMing Wang et al., [5] proposed a developing DBN with exchange learning (TL-GDBN) consequently choose its structure estimate, which can quicken its learning procedure and enhance demonstrate exactness. DBN is made out of various limited Boltzmann machines (RBMs) stacked consecutively. Its learning procedure can be separated into two phases: unsupervised getting the hang of (pretraining) and administered adapting (fine-tuning).

Chaolong Zhang et al., [6] proposed strategy utilizes an avaricious layer-wise preparing model in its relating stage initially, and then embraces a back-engendering in consequent fine-tuning stage. In the work, DBN is used to remove proficient highlights for simple circuit nascent blame analysis.

Jiaqi Xie et al., [7] proposed a start to finish blame determination show dependent on a versatile DBN improved 
by the Nesterov minute (NM) to extricate deep delegate highlights from pivoting apparatus and perceive bearing deficiency types and degrees all the while. Recurrence space signals are inputted into the model for highlight learning, and $\mathrm{NM}$ is acquainted with the preparation procedure of the DBN demonstrate. Individual versatile learning rate calculations are then connected to enhance parameter refreshing. The execution of the proposed technique is approved utilizing an independent bearing deficiency test stage, and the model is appeared to accomplish agreeable assembly and a testing exactness higher than those got from standard DBN and bolster vector machine. The diverse creator sees on the writing overview mostly centered around the DBN based highlights extraction technique incorporates two preparing stages: pre-preparing stage and fine-tuning stage. In the pre preparing stage, the information flag information are learnt by the primary Restricted Boltzmann Machine (RBM) and the principal layer's highlights are created. The proposed work depict in the section3.

\section{PROPOSED METHOD FOR EYE DISEASES DETECTION}

\section{A. Image pre-processing}

Pre-preparing is a typical name for tasks with images at the most minimal dimension of deliberation for both input information and output are intensity images The point of pre-handling is an enhancement of the image information that remove undesirable distortions or improves some image highlights critical for further preparing. The noise occurred in the image can be filtered through the procedure. The resize the image can be satisfying for the further process [8] and [9]. The calculation steps are as per the following.

Algorithm for eye skin detection

Step1: Eye Image database

Detection of red eye skin defects in photographic images Step2: Image Denoising
The Gaussian noise, Salt and Pepper noise, Speckle noise and Poisson noise filtered in image denoising.

Step3: Image segmentation

The image segmentation method applied to separate the red skin portion in an eye.

Step4: Detection process

Red skin execution valueWRSA $=\frac{\varepsilon W p+\varphi W B}{\varepsilon+\varphi}$

Step5: Determine skin value

$\mathrm{WP}=\frac{\alpha W H+\beta W k 0}{\alpha+\beta}$

Step6: Determination of contrast value

$\mathrm{WB}=\frac{\gamma \mathrm{W} 0+\delta \mathrm{Wkv}}{\gamma+\delta}$

Step7: Determination of high resolution image context WRSA $=<17 \%$

Step8: Estimate the high resolution image content

The eye image database were taken as the info image, the red skin present in the identified utilizing the given calculation. The means associated with the procedure to remove the commotion present in the image. The segmentation part isolates the typical eye divide and the detected eye partition. The edge identification depends on the recognition procedure, skin value, and contrast value. The high resolution image segment can be distinguished of having the red skin defected portion include the incentive inside the range of $17 \%$. The figure 1 demonstrates the segmentation partitions an image into particular districts containing every pixel with similar properties of red skin value utilizing eye skin identification algorithm. In the proposed work, the segmentation focuses on the finding the edges of the influenced portion present in the image. To be important and helpful for image examination and elucidation, the locales ought to unequivocally identify with portrayed articles or highlights of intrigue. Significant segmentation is the initial step from low-level image processing transforming a grayscale or color image into one or more different images to abnormal state image portrayal as far as highlights, articles, and scenes.

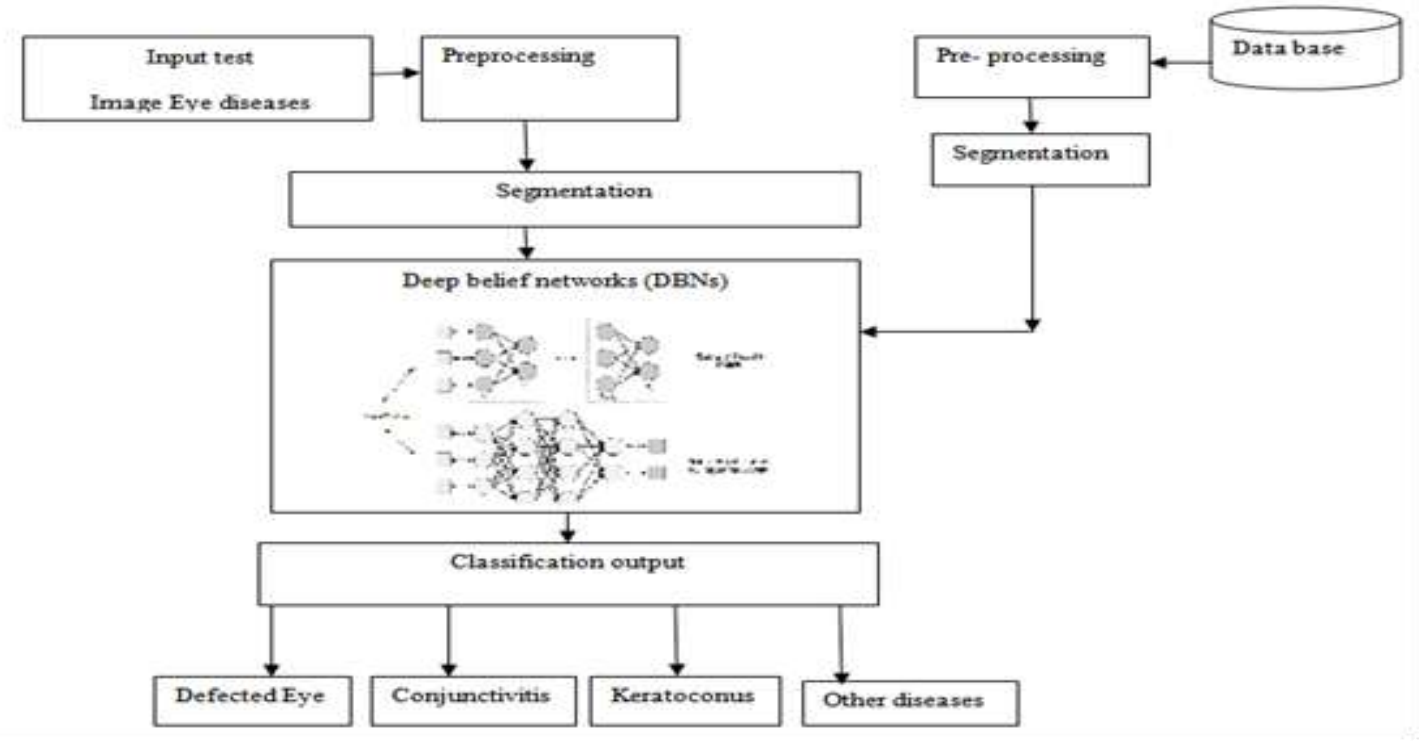

Figure 1: Eye diseases classification method. 
The accomplishment of image examination relies upon unwavering quality of segmentation; however a precise apportioning of an image is done through the classifier utilized. The recognized district is given as the contribution for a canny network utilizing a Deep Belief Network.

\section{B. Deep Belief Network}

The DBN connections are downward direction to the side of the next lower layer expect that the upper two layers are in single direction. A DSN needs a hybrid model consists of the

first two layers were unidirected graphical model. The remaining work needs a directed generative model. The various distinctive layers are found out in a layer shrewd greedy fashion and fine-tuned dependent on required yield be that as it may, the preparation method is computationally demanding. The distinctive layers are found out in a layer shrewd greedy fashion and fine-tuned dependent on required yield be that as it may, the preparation method is computationally demanding. The Unsupervised Probabilistic

Deep learning algorithm used in the DBN. The DBN id composed of various multi layers of latent variables of stochastic in nature. The binary form of latent variables used as the feature detectors. The another way of hidden units in DBN were designed for hybrid graphical model. The top two layers were undirected and also the lower layers needs the direct connection obtained from the upper layer.

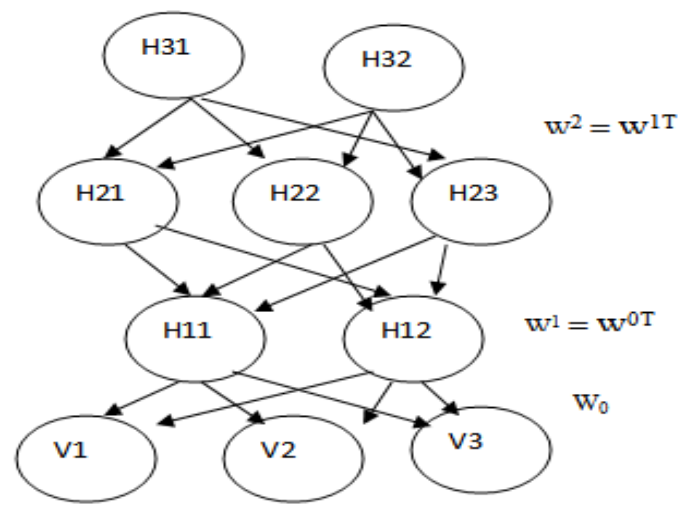

Figure 2 Deep Belief Network

The proposed work uses the Restricted Boltzmann Machine (RBM) in the form of stack. The two layers of DBN link with the connections based on the undirected, symmetric connection along with the memory allocated in the work. The associations between all lower layers are coordinated, with the bolts indicated the layer that is nearest to the information. Lower Layers have coordinated a cyclic association that converts affiliated memory to watched factors. The most reduced layer or the unmistakable units gets the info information. Info information can be twofold or genuine. There are no intra layer associations likes RBM. Hidden units speak to highlights that catch the relationships present in the information. Two layers are associated by a network of symmetrical loads W. Each unit in each layer is associated with each unit in the each neighboring layer. Learning, the estimations of the dormant factors in each layer can be derived by a solitary, base up pass. Greedy pretraining begins with a watched information vector in the base layer. It at that point utilizes the generative loads in the turn around bearing utilizing adjusting. The proposed work utilizes the DBN for preparing the references information taken from the information base after pre-handling and division process happens. The DBN has prepared qualities both the information picture and the database picture. The information got from the both the info picture and the database is gone through the classifier and the yield were analyse [10] and [11]. The design of DBN makes it conceivable to digest higher dimension includes through layer adaptation. Each layer of hidden factors figures out how to speak to highlights that catch higher order correlations in the original input information. Applying DBNs to an order issue, highlight vectors from information tests are utilized to set the conditions of the unmistakable factors of the lower layer of the DBN. The DBN is then prepared to deliver a likelihood conveyance over the conceivable names of the information dependent on back likelihood dissemination of the information tests. The proposed work made out of the data set taken from the input image $S=\{\{x 1, y 1\},\{x 2, y 2\}, \ldots,\{x N$ , $\left.\mathrm{yN}_{\text {N }}\right\}$ contains an all-out number of $\mathrm{N}$ information test sets $\{x n, y n\}$, where $x n$ is the nth information test, yn is the comparing nth target name. Expect a DBN comprises of $\mathrm{H}$ shrouded layers and the parameters of each layer $I \in\{1, \ldots$, $\mathrm{HH}\}$ by $\theta \mathrm{i}=\{\mathrm{Wi}$, bi $\}$. Given an info information test $\mathrm{x}$ from the informational collection, the DBN with $\mathrm{HH}$ concealed layer(s) presents an intricate element mapping capacity. After component change, delicate max layer fills in as the yield layer of DBN to perform grouping forecasts as parameterized by $\theta \mathrm{s}=\{\mathrm{Ws}$, bbs $\}$. Assume there are $\mathrm{K}$ neurons in the softmax layer, where the $\mathrm{j}$ th neuron is in charge of evaluating the forecast likelihood of class $j$, given contribution of $\mathrm{xH}$ which is the yield of the past layer

and connected with loads $\mathrm{W}(\mathrm{j}) \mathrm{s}$ and predisposition $\mathrm{b}(\mathrm{j})$

$$
P\left(y=\frac{j}{x}\right)=\frac{\exp \left(b b_{S}^{j}+X_{H H}^{T} W_{S}^{(j)}\right.}{\sum_{k=l}^{k} \exp \left(b b_{S}^{(k)}+X_{H}^{T} W_{S}^{(k)}\right.}
$$

where $\mathrm{xH}$ is the yield of the past layer. In light of the likelihood estimation, the prepared DBN classifier gives an expectation as

$$
f(x)=\operatorname{argmax}_{1 \leq j \leq k} \mathrm{p}(\mathrm{y}=j / x)
$$

By and by, the parameters $\{\theta 1, \theta 2, \ldots, \theta \mathrm{H}, \theta \mathrm{s}\}$ of DBN are hugely enhanced by statistic slope descent as for the negative log-probability misfortune over the training set $\mathrm{St}$. The yield of the DBN characterizes the influenced eye infections might be the Keratoconus, conjunctivitis, ordinary defected eye or it might be different ailments.

\section{Architecture for Restricted Boltzmann Machines}

A RBM is a MRF related with a bipartite undirected chart. It comprises of $\mathrm{m}$ unmistakable units $\mathrm{V}=(\mathrm{V} 1, \ldots, \mathrm{Vm})$ to speak to recognizable information and $\mathrm{n}$ concealed units $\mathrm{H}=$ $(\mathrm{H} 1, \ldots, \mathrm{Hn})$ to catch conditions between watched factors. In parallel RBMs, our concentration in this instructional exercise, the irregular factors $(\mathrm{V}, \mathrm{H})$ take esteems $(\mathrm{v}, \mathrm{h}) \in\{0$, $1 \mathrm{~m}+\mathrm{n}$ and the joint likelihood dissemination under the model is given by the Gibbs circulation with the vitality work appeared in condition. 


$$
\begin{aligned}
& p(v, h)=\frac{1}{z} e^{-E e(v, h)} \\
& E(v, h)= \\
& -\sum_{i i=1}^{n} \sum_{j=1}^{m} w_{i j} h_{i i} V_{j j}-\sum_{j=1}^{m} b_{i i} V_{j j}-\sum_{j=1}^{m n} c_{i i} h i
\end{aligned}
$$

For all $\mathrm{ii} \in\{1, \ldots, \mathrm{n}\}$ and $\mathrm{jj} \in\{1, \ldots, \mathrm{m}\}$, wij is a genuine esteemed weight related with the edge between units $\mathrm{Vjj}$ and Hii and bjj and cii are genuine esteemed inclination terms related with the jth The classifier designed based on the graph of a RBM has just associations between the layer of covered up and noticeable factors yet not between two factors of a similar layer. As far as likelihood this implies the concealed factors are free given the condition of the obvious factors and the other way around. Obvious and the ith shrouded variable, individually.

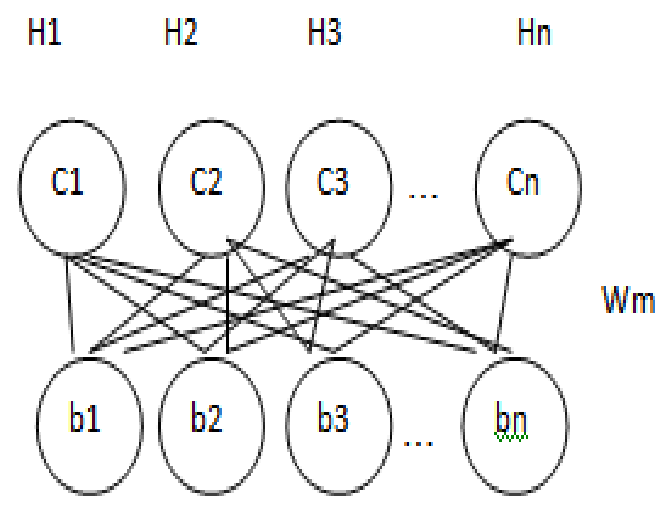

Figure 3: The undirected graph of an RBM with $n$
hidden and $m$ visible variables

The RBM can be deciphered as a stochastic neural system, where hubs and edges correspond to neurons and synaptic associations, individually. The restrictive likelihood of a solitary variable being one can be deciphered as the terminating rate of a (stochastic) neuron with sigmoid initiation work.

$$
\begin{aligned}
& \sigma\left(x_{i}\right)=\frac{1}{\left(1+e^{-x i}\right)} \\
& p\left(H_{i}=\frac{1}{v}\right)=\sigma\left(\sum_{j=1}^{m} w_{i j} v_{j}+c_{i}\right. \\
& p\left(V=\frac{1}{h}\right)=\sigma\left(\sum_{i=1}^{n} w_{i j} h_{j}+b_{i}\right.
\end{aligned}
$$

The autonomy between the factors in a single layer makes Gibbs sampling particularly simple: Instead of inspecting new qualities for all factors in this way, the conditions of all factors in a single layer can be tested mutually. Therefore, Gibbs inspecting can be performed in only two sub steps: examining another state $h$ for the concealed neurons dependent on $\mathrm{p}(\mathrm{h} \mid \mathrm{v})$ and testing a state $\mathrm{v}$ for the obvious layer dependent on $\mathrm{p}(\mathrm{v} \mid \mathrm{h})$. This is likewise alluded to as square Gibbs sampling.

\section{IV.PERFORMANCE EVALUATION \& RESULTS}

The execution of the system can have a characterization assignment, the exactness for a class is the number of genuine positives (for example the quantity of things effectively marked as having a place with the positive class) separated by the all-out number of components named as having a place with the positive class (for example the whole of genuine positives and false positives, which are things erroneously marked as having a place with the class).
Review in this context is characterized as the quantity of genuine positives separated by the all out number of components that really belong to the positive class (for example the entirety of genuine positives and false negatives, which are things which were not named as having a place with the positive class yet ought to have been).The trial result were broke down in the from $\mathrm{P}$ positive examples and $\mathrm{N}$ negative occurrences for some condition.

Precision and recall are then defined as

$$
\begin{aligned}
& \text { Recall }=\frac{t p}{t p+f n}=\frac{42}{42+13}=0.76 \\
& \text { Precision }=\frac{t p}{t p+f p}=\frac{42}{42+16}=0.724
\end{aligned}
$$

The $t p, t n, f p, f n$ were referred to as a true positives (tp), true negatives(tn), false positives(fp), and false negatives (fn).

$$
\begin{aligned}
& \text { True negative rate }=\frac{t n}{t n+t p} \\
& \text { Accuracy }=\frac{t p+t n}{t p+t n+f p+f n}
\end{aligned}
$$

The human eyes with different kinds of images are appeared in figure4. The reddish surface of the eyes is same for every one of the types of images yet they are having the varied diseases.

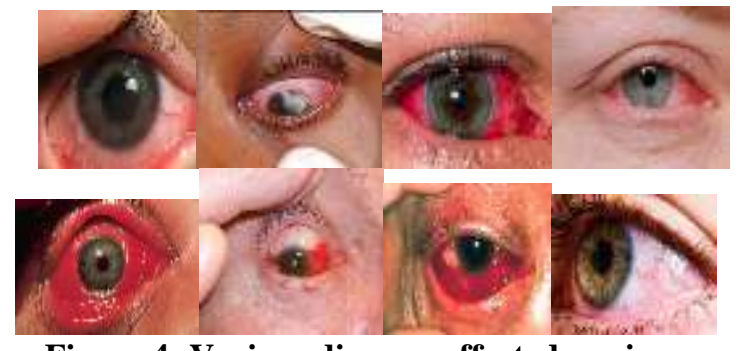

Figure 4: Various diseases affected eye image.

The reddish types of images are taken for the analysis. Here the implementation based on the various types of eye images is taken for the analysis. The colour image is converted into gray scale images of the same size of $256 \mathrm{x}$ 256. The wiener filter is used for the removing majority of noise present in the image. The segmentation process segment the image based on the intensity of the gray scale images. The eye skin detection algorithm extracts the features of the intensity of the pixel present in the image. The DBN classify the input test values with the Keratoconus the simulation results are shown below.

\begin{tabular}{|l|l|l|}
\hline $\begin{array}{l}\text { Threshold } \\
=0.5\end{array}$ & $\begin{array}{l}\text { Actual } \\
\text { positives }\end{array}$ & Actual Negativies \\
\hline $\begin{array}{l}\text { Predicted } \\
\text { positives }\end{array}$ & $42(\mathrm{TP})$ & $16(\mathrm{FP})$ \\
\hline $\begin{array}{l}\text { Predicted } \\
\text { Negatives }\end{array}$ & $13(\mathrm{FN})$ & $29(\mathrm{TN})$ \\
\hline
\end{tabular}

Table 1 Performance values.

$$
\begin{aligned}
& F 1 \text { score }=2 * \frac{\text { precision } * \text { recall }}{\text { precision }+ \text { recall }}=0.74 \\
& \text { False positive rate }=\frac{F p}{F p+T n}=\frac{16}{16+29}=0.39 \\
& \text { True positive rate }=\frac{F p}{F p+T n}=\frac{42}{42+13}=0.76
\end{aligned}
$$




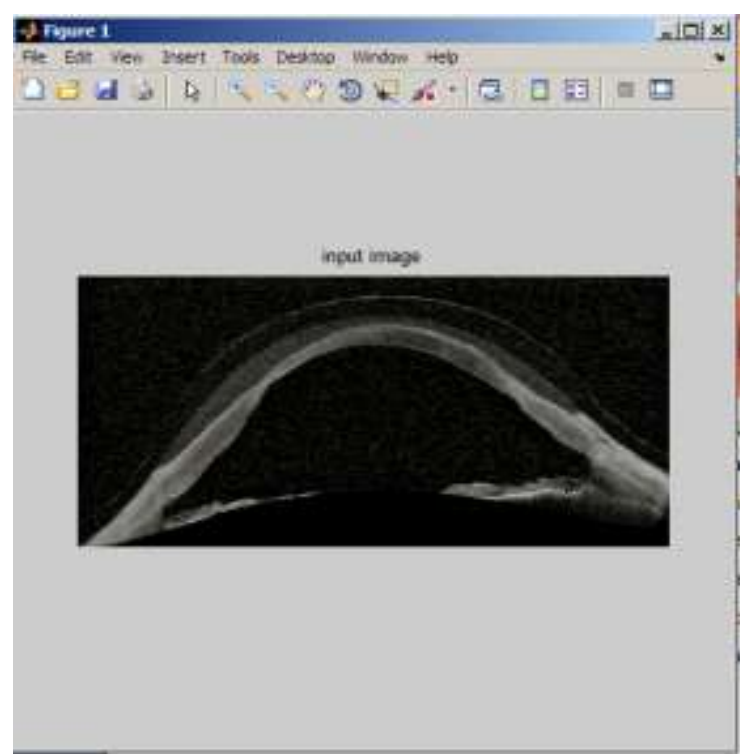

5.a) Input grayscale eye image

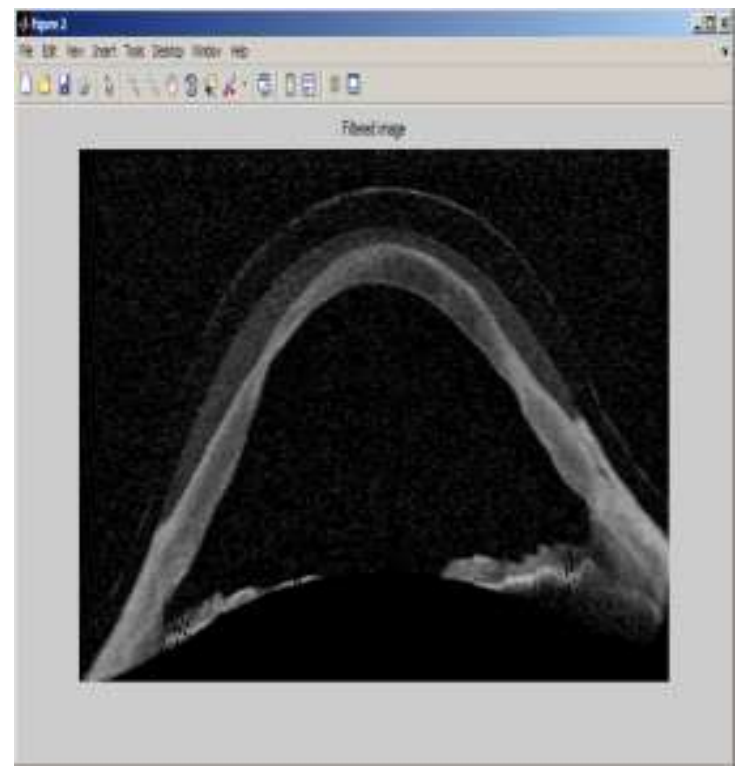

5.b) filter image using wiener filter

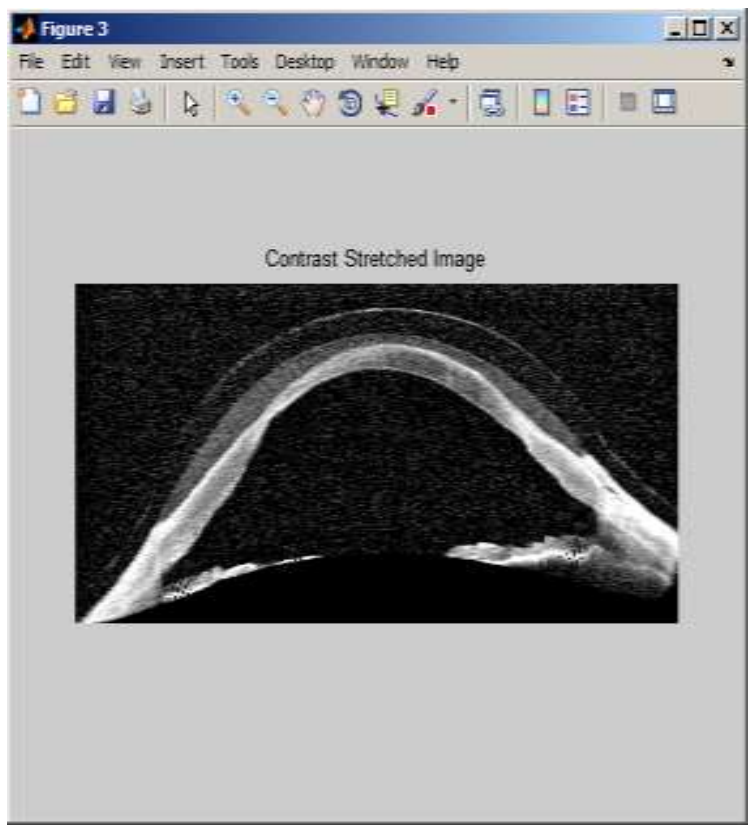

5.c Contrast enhanced image

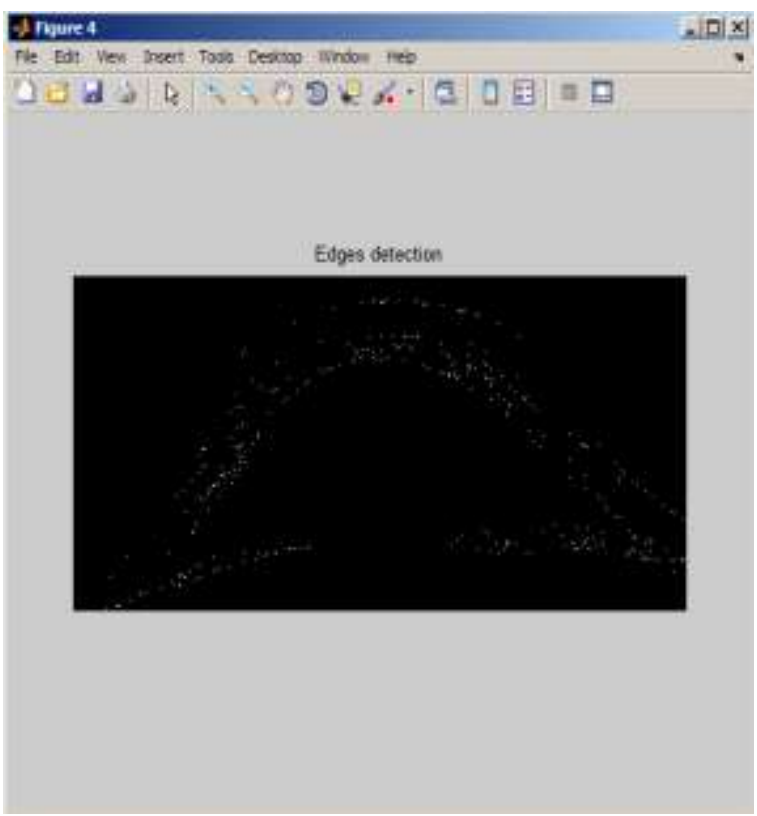

\section{5.d Edge detection output}

The figure 5.a shows the input gray scale image, the image filtered using wiener filter shown in the figure 5.b. The contrast is enhancement is shown in the figure 5.c. The edge detection process is shown in the figure 5.d.The result of the classification process shows the input image is Keratoconus diseased eye image.

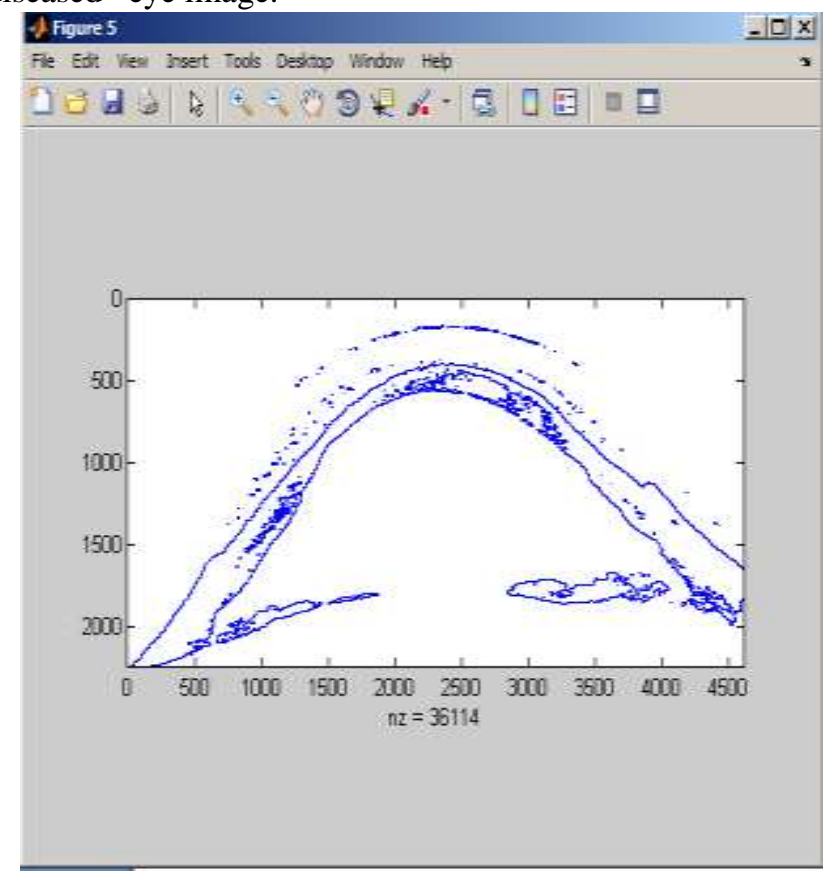

5.e Keratoconus image

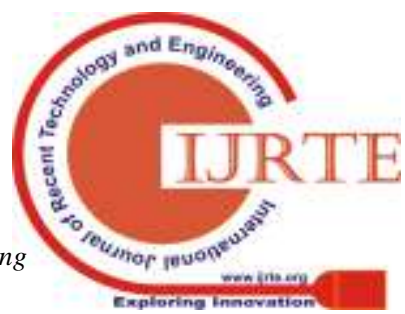




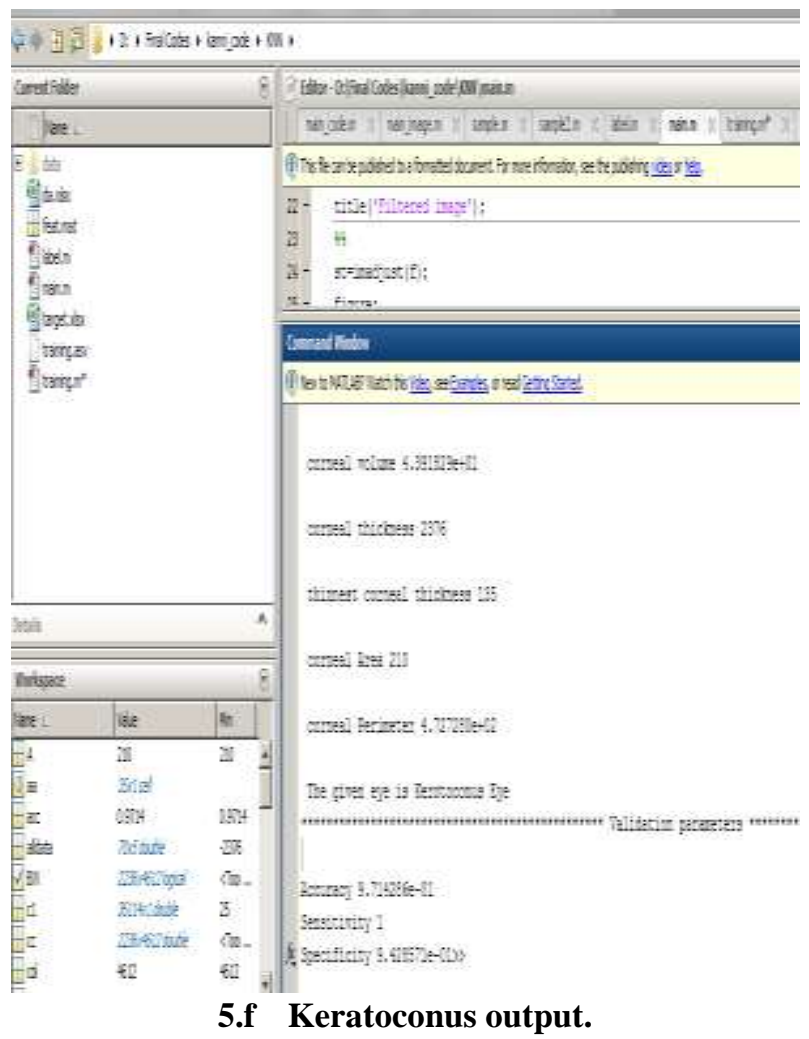

Figure 5. The result of the proposed system.

The output of the classifier shown here is the Keratoconus affected input eye image. The sensitivity value of the image is 1 and the accuracy of the classifier is $95.23 \%$.

\section{CONCLUSION}

The human eye diseases can be detected and classified based on the Deep - belief network (DBN) based on the image processing techniques. The preprocessing, image segmentation, classifications are the image processing techniques used in the processes. The classifier able to classify the eye diseases like the conjunctivitis, Keratoconus, defected eyes and other diseases. The performance of the classifier can be estimated using accuracy, precision and recall value were $95.23 \%, 0.76,0.74$.

\section{REFERENCES}

1. Mufti Mahmud, Senior Member, IEEE, Mohammed Shamim Kaiser, Senior Member, IEEE, Amir Hussain, Senior Member, IEEE, and Stefano Vassanelli," Applications of Deep Learning and Reinforcement Learning to Biological Data", IEEE TRANSACTIONS ON NEURAL NETWORKS AND LEARNING SYSTEMS, Digital Object Identifier 10.1109/TNNLS.2018.2790388.

2. Siqi Nie, Meng Zheng, and Qiang Ji," The Deep Regression Bayesian Network and Its Applications", IEEE Signal Processing Magazine | January 2018 |, Digital Object Identifier 10.1109/MSP.2017.2763440.

3. Manuel Titos, Angel Bueno, Luz Garc'1a, and Carmen Ben'itez," A Deep Neural Networks Approach to Automatic Recognition Systems for Volcano-Seismic Events", IEEE JOURNAL OF SELECTED TOPICS IN APPLIED EARTH OBSERVATIONS AND REMOTE SENSING, Digital Object Identifier 10.1109/JSTARS.2018.2803198.

4. Yi Qin, Member, IEEE, Xin Wang, Jingqiang Zou," The optimized deep belief networks with improved logistic
Sigmoid units and their application in fault diagnosis for planetary gearboxes of wind turbines," IEEE TRANSACTIONS ON INDUSTRIAL ELECTRONICS, DOI 10.1109/TIE.2018.2856205.

5. GongMing Wang, Student Member, IEEE, JunFei Qiao, Member, IEEE, Jing Bi , Senior Member, IEEE,

WenJing Li, and MengChu Zhou , Fellow, IEEE," TL-GDBN: Growing Deep Belief Network With Transfer Learning", IEEE TRANSACTIONS ON AUTOMATION SCIENCE AND ENGINEERING, Digital Object Identifier 10.1109/TASE.2018.2865663.

6. CHAOLONG ZHANG 1,2, YIGANG HE2, LIFENG YUAN 3, AND SHENG XIANG3," Analog Circuit Incipient Fault Diagnosis Method Using DBN Based Features Extraction", IEEE Access, Digital Object Identifier 10.1109/ACCESS.2018.2823765.

7. JIAQI XIE1, GUIFU DU 1, CHANGQING SHEN 1,2, NAN CHEN 2, (Member, IEEE), LIANG CHEN1, AND ZHONGKUI ZHU," An End-to-End Model Based on Improved Adaptive Deep Belief Network and Its Application to Bearing Fault Diagnosis", Received September 25, 2018, accepted October 16, 2018, date of publication October 23, 2018, date of current version November 19, 2018. Digital Object Identifier 10.1109/ACCESS.2018.2877447.

8. Chong Zhang, Kay Chen Tan , Fellow, IEEE, Haizhou Li , Fellow, IEEE, and Geok Soon Hong," A Cost-Sensitive Deep Belief Network for Imbalanced Classification", IEEE TRANSACTIONS ON

NEURAL NETWORKS AND LEARNING SYSTEMS, Digital Object Identifier 10.1109/TNNLS.2018.2832648.

9. A. M. Nickfarjam, H. Ebrahimpour-komleh," Multi-input Topology of Deep Belief Networks for Image Segmentation", 978-1-4673-9762-9/15/\$31.00 (02015 IEEE.

10. Seong-Wook Park, Member, IEEE, Junyoung Park, Student Member, IEEE, Kyeongryeol Bong, Member, IEEE, Dongjoo Shin, Member, IEEE, Jinmook Lee, Student Member, IEEE, Sungpill Choi, Member, IEEE, and Hoi-Jun Yoo, Fellow, IEEE," An Energy-Efficient and Scalable Deep Learning/Inference Processor With Tetra-Parallel MIMD Architecture for Big Data Applications, IEEE TRANSACTIONS ON BIOMEDICAL CIRCUITS AND SYSTEMS ,Digital Object Identifier 10.1109/TBCAS.2015.2504563.

11. Chong Zhang, Pin Lim, A. K. Qin, Senior Member, IEEE, and Kay Chen Tan, Fellow, IEEE," Multiobjective Deep Belief Networks Ensemble for Remaining Useful Life Estimation in Prognostics", IEEE TRANSACTIONS ON NEURAL NETWORKS AND LEARNING SYSTEMS, Digital Object Identifier 10.1109/TNNLS.2016.2582798. 